\title{
Relative body fat and anthropometric prediction of body density of female athletes*
}

\author{
R. T. Withers, N. O. Whittingham, K. I. Norton, J. La Forgia, M. W. Ellis, and A. Crockett \\ The Exercise Physiology Laboratory, School of Education, The Flinders University of South Australia, Bedford Park, \\ South Australia 5942
}

Eur J Appl Physiol (1987) 56:169-180

During the final printing process the following two corrections were unfortunately not carried out:

1. On page 169, line 27 of the Summary, the first "of" should have been deleted.

2. On page 177 , line 8 of the right-hand column, "for an equation" should have been deleted. 